\title{
Leiomyoma of the Male Urethra
}

\section{Kwang Woo Lee}

Departments of Urology, Soonchunhyang University Bucheon Hospital, Soonchunhyang University College of Medicine, Bucheon, Korea

\begin{abstract}
Leiomyomas are benign tumors of smooth muscle origin that can occur at any location in the urinary tract. The kidney capsule is the most common location in the genitourinary tract. Leiomyomas of the urethra are a very rare entity; they are more frequent in females than in males. Only eight cases leiomyomas of the male urethra have been reported in the English-language medical literature. We report one additional case of pedunculated leiomyoma originating from the posterior wall of the male prostate urethra; this tumor appeared to be a bladder tumor on computed tomography.
\end{abstract}

Keywords: Leiomyoma; Urethra; Male

\section{INTRODUCTION}

Primary tumors rarely develop in the urethra. The majority of the tumors are malignant and most tumors are carcinomas [1]. In addition, it has been reported that both malignant and benign tumors occur more frequently in premenopausal females in their forties [2]. Urethral leiomyomas originate from the smooth muscle. In 1995, Bartolozzi et al. [3] reported more than 110 cases of urethral leiomyomas. However, this tumor has been reported only in eight male patients. Although leiomyomas are rare, they are the most common tumor, among the mesenchymal neoplasms that develop in the urethra. Leiomyomas develop mainly from the circular fibers of the smooth muscle and can develop throughout the genitourinary system. However, the kidney capsule is the most common location. We report herein the case of a 35-year-old man with a urethral leiomyoma that developed in the prostatic urethra and review of the literature.

\section{CASE REPORT}

A 35-year-old man presented with a bladder mass that was incidentally detected by computed tomography (CT) of the abdomen, performed as part of a health-screening program. The patient had a 3-year history of microscopic hematuria on urinalysis without any special treatment. He had no history of smoking and his family and social histories were unremarkable. The patient did not have any voiding problem and the physical examination revealed a good general condition and stable vital signs. The laboratory data, including routine hematologic tests, hematochemical tests, and immunoserological tests, exhibited normal values. There was no microscopic hematuria on urinalysis. The urine cultures and urine cytology were negative.

$\mathrm{CT}$ of the abdomen revealed a mass measuring approximately $1.6 \mathrm{~cm}$ in diameter that protruded into the bladder from the midline of the posterior wall of the bladder base (Fig. 1A). The mass was enhanced in the arterial phase, but neither peripheral fat infiltration nor enlargement of the pelvic and inguinal lymph nodes was observed (Fig. 1B). CT of the abdomen established the diagnosis of a primary bladder tumor and transurethral resection of the tumor was performed.

When the resectoscope was introduced into the urethra, a 2.0$\mathrm{cm}$ narrow, linear stalk connecting the mass and the posterior wall of the prostatic urethra, which was located left of the verumontanum, was noted. A pedunculated mass with a roundshaped lesion $(1.7 \times 1.1 \times 0.9 \mathrm{~cm})$ on its top was identified. The mass had a smooth surface without erosions. There were no adhe-
(C) 2016 Soonchunhyang Medical Research Institute This is an Open Access article distributed under the terms of the Creative Commons Attribution Non-Commercial License (http://creativecommons.org/licenses/by-nc/4.0/). 

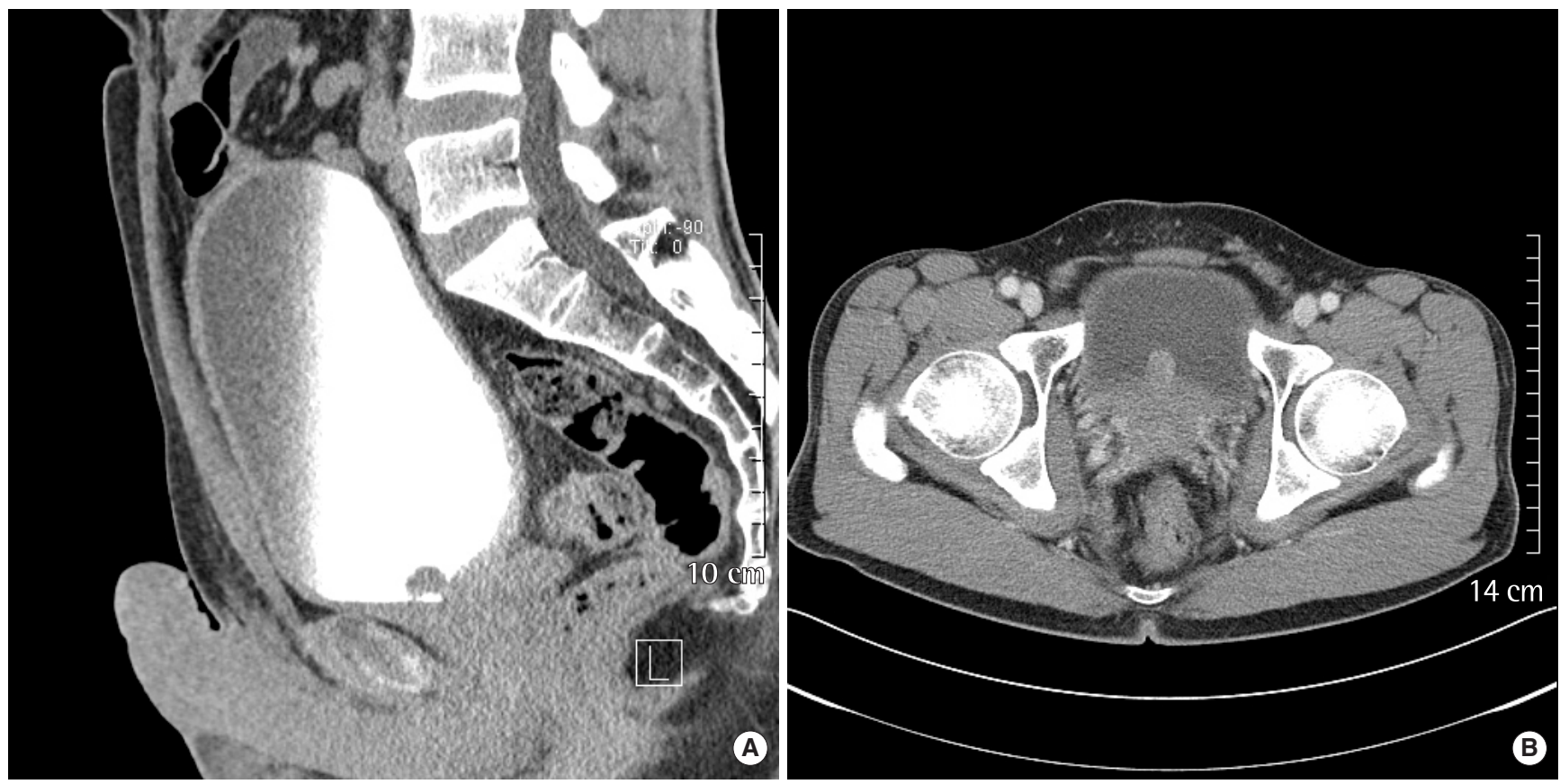

Fig. 1. (A) Computed tomography of the pelvis shows a well-defined lobulated contoured mass in inferior portion of the bladder. (B) Computed tomography of the pelvis shows a $1.6 \times 1.5 \mathrm{~cm}$ enhancing mass in the posterior wall of the bladder during the arterial phase, with no evidence of perivesical fat infiltration and pelvic lymph node invasion.
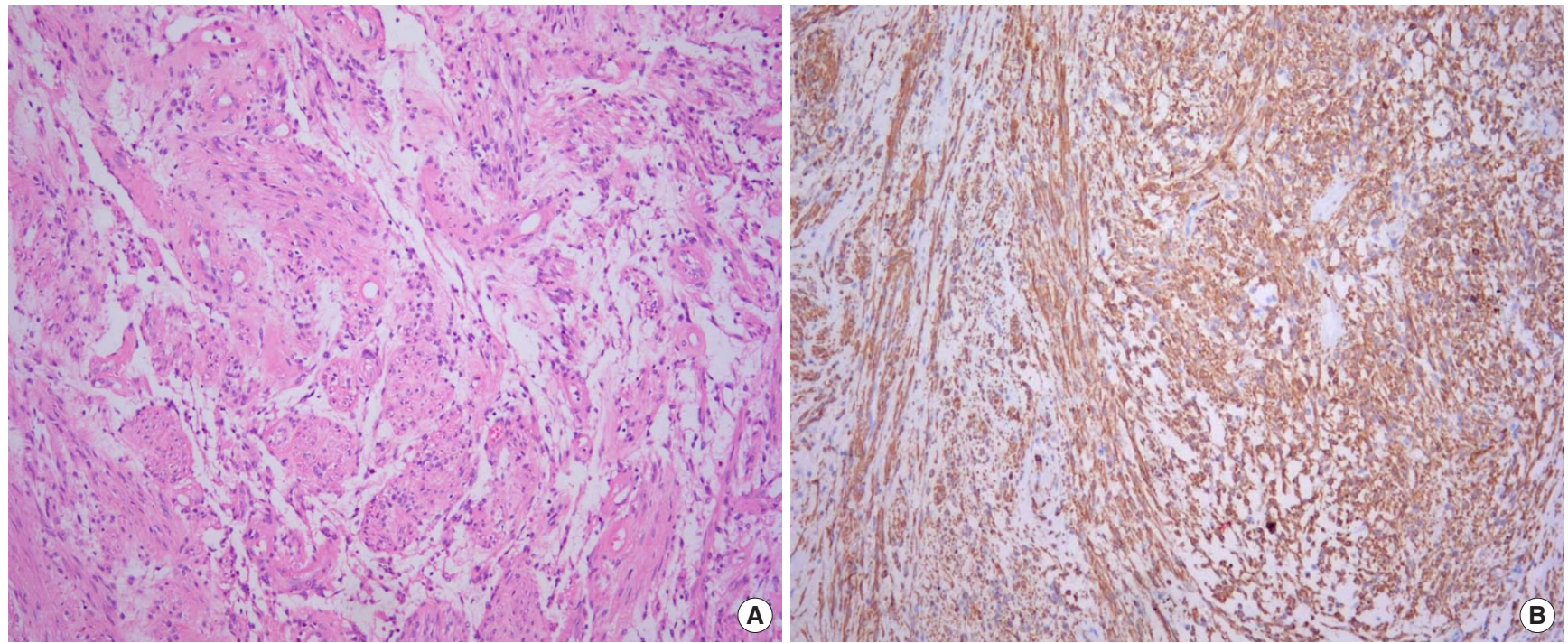

Fig. 2. (A) Photomicrograph showing intersecting bundles of smooth muscle cells $(H \& E, \times 200)$. (B) Immunohistochemical stain for smooth muscle actin shows a strong positive reaction in the spindle tumor cells $(\times 100)$.

sions between the mass and the bladder wall except for the stalk. The tumor was resected through the urethra after dissecting its base from the prostatic urethra.

Pathology revealed bundles of spindle cells in the myxoid stroma under the urothelium and focally intersecting bundles of smooth muscle cells (Fig. 2A). Immunochemistry showed a posi- tive reaction to actin and desmin of the smooth muscle (Fig. 2B), which supported the diagnosis of urethral leiomyoma. The patient was discharged from the hospital without specific complications 1 day after surgery. At 2 years of follow-up, cystoscopy findings were normal. 


\section{DISCUSSION}

Urethral leiomyomas are classified as mesenchymal tumors [4]. Although urethral leiomyomas are rare, it is the most common mesenchymal neoplasm of the urethra and occurs more commonly in females than in males. There have been many reports of this tumor in females. However, there have been only eight cases reported in males in the English-language literature [5].

Urethral leiomyomas originate from the circular fibers of the smooth muscle, but its pathogenesis has not yet been elucidated. Shield and Weiss [6] have indicated that urethral leiomyomas are associated with estrogen levels based on the fact that the majority of cases with this tumor show rapid growth during pregnancy; this supports its high estrogen sensitivity; whereas the size of this tumor decreases after menopause. However, it rarely develops in young males or postmenopausal women. It is likely that there are multifactorial determinants involved in its pathogenesis.

Urethral leiomyomas develop most commonly in the proximal urethra, particularly the posterior wall of the urethra [4,7]. In the literature on leiomyomas of the male urethra, six cases developed in the bulbous urethra, one case in the penile urethra, and one case in the prostatic urethra [5]. Our case developed in the prostatic urethra.

The clinical manifestations generally include a painless palpable mass, abnormal sexual sensations, dyspareunia, incontinence, frequency, and urinary tract infections. Mooppan et al. [2] have reported that urinary tract infections are the most common presenting problems. Urethral leiomyomas may occur without any symptoms or may manifest as a painless palpable mass on the anterior wall of the vagina. It can develop throughout the genitourinary system. Although it develops most commonly in the kidneys, there have been several reports of such tumors in the bladder, prostate, penis, scrotum, epididymis, and spermatic cord. The size of most urethral leiomyomas has been reported to range between 1 and $8 \mathrm{~cm}$; however, one exceptional case was reported with a 40 cm leiomyoma [2].

The differential diagnosis includes caruncle, diverticulum, ure- thral prolapse, papilloma, ectopic ureterocele, fibrous polyp, malignant lymphoma, and leiomyosarcoma. Histopathologically, in contrast to leiomyosarcoma, which exhibits atypical cells, pleomorphism, and differentiation of multiple cell types, the leiomyoma reveals intersecting bundles of smooth muscle cells in the myxoid stroma with the absence of atypical cells and pleomorphism. In addition, leiomyomas show a strong positive response to actin and desmin smooth muscle antibodies on immunochemical staining.

Cystourethroscopy, urethrography and CT scan can be used for the diagnosis of a urethral leiomyoma, but it is difficult to identify its exact location. Magnetic resonance imaging is useful in confirming the exact location of this tumor. In cases of urethral leiomyomas with erosions or ulcerations on their surface, histopathological examination is most helpful in the differentiation from malignant tumors. Leiomyomas can be successfully treated with regional excision or transurethral resection. There have been no reports of malignant transformation. The clinical outcome in patients with a urethral leiomyoma has been reported to be excellent; there is only one female case of recurrence reported [8]. Our patient showed no recurrence during a follow-up period of 2 years after surgery.

\section{REFERENCES}

1. Mira JL, Fan G. Leiomyoma of the male urethra: a case report and review of the literature. Arch Pathol Lab Med 2000;124:302-3.

2. Mooppan MM, Kim H, Wax SH. Leiomyoma of the female urethra. J Urol 1979;121:371-2.

3. Bortolozzi G, Grasso A, Zasso B. Leiomyoma of the female urethra: a case report and review. Clin Exp Obstet Gynecol 1995;22:169-71.

4. Saad AG, Kaouk JH, Kaspar HG, Khauli RB. Leiomyoma of the urethra: report of 3 cases of a rare entity. Int J Surg Pathol 2003;11:123-6.

5. Garrido Abad P, Fernandez Arjona M, Herranz Fernandez LM, MunozDelgado Salmeron J, Capote LF. Leiomyoma of the male urethra: case report and review of the literature. Arch Esp Urol 2010;63:71-4.

6. Shield DE, Weiss RM. Leiomyoma of the female urethra. J Urol 1973;109: 430-1.

7. Wani NA, Bhan BL, Guru AA, Garyali RK. Leiomyoma of the female urethra: a case report. J Urol 1976;116:120-1.

8. Lake MH, Kossow AS, Bokinsky G. Leiomyoma of the bladder and urethra. J Urol 1981;125:742-3. 